\title{
An observational study of alopecia areata in Sri Lankan adult patients
}

\author{
R R Ranawaka
}

\begin{abstract}
Objectives The objectives were to assess the demographical pattern, clinical presentation and therapeutic response in a cohort of patients with alopecia areata (AA) in Sri Lanka.
\end{abstract}

Methods Hospital-based observational study of 290 adults aged 18 years or above.

Results Alopecia areata was commoner in men (M:F=1.3:1). Age of onset was between 20-35 years (median 31 years) in $61 \%$. Those with juvenile-onset $A A(\leq 17$ years, $\mathrm{n}=5)$ showed severe disease with many relapses and resistance to therapy. Late-onset $A A(<50$ years, $n=12$ ) was commoner among females and had mild disease activity. Alopecia areata was the commonest clinical type $(93.7 \%)$, followed by alopecia universalis $(n=10)$, ophiasis pattern $(n=3)$, alopecia totalis $(n=3)$, and reverse ophiasis pattern $(n=1)$. Mild disease ( $>10 \%$ scalp area) was the commonest (82\%). Alopecia was total, universal or extensive $(>10 \%$ scalp area) in $18 \%$. Sites involved were scalp $(71 \%)$, beard only $(20.5 \%)$ and multiple sites (8.7\%). Nail changes were associated with severe disease. Associated autoimmune diseases were vitiligo $6(2 \%)$, thyroid disease $5(1.7 \%)$ and rheumatoid arthritis $1(0.3 \%)$. Atopy $(21 \%)$ was not associated with younger age of onset or severity of disease. Patients with a family history among first degree relatives had earlier onset of disease.Most (61\%) were cured after 1-2 intralesional steroid injections. Oral dexamethasone mini pulse with or without topical $5 \%$ minoxidil lotion for 12 months or more were used in $28 \%$.

Conclusions In Sri Lanka AA is a disease of the young. Extensive disease, juvenile onset, and associated nail changes were poor prognostic factors.

Ceylon Medical Journal 2014; 59: 128-131

\section{Introduction}

Alopecia areata (AA) is a common, non-scarring, patchy loss of hair in the scalp and elsewhere [1-4]. Onset of AA may occur at any age and peaks between the second and fourth decades [4]. The sex incidence is equal [4]. But male preponderance has been reported from Turkey and India [2, 3]. Its pathogenesis is uncertain, however, auto- immunity has been suggested [5, 6]. But environmental factors such as infection and psychological stress may play an important role [5].

Familial incidence of AA is $10-20 \%$, but in monozygotic twins it is 50\% [7]. An association between AA and atopic disease has been identified. Those with associated atopic disease have earlier age of onset and more severe disease than non-atopic subjects [7]. This association has been disputed in studies from Singapore and India [1,2].

The frequency of other associated diseases are; thyroid disease $2.3 \%$, vitiligo $4.1 \%$, diabetes mellitus $3.2 \%$, Down's syndrome $1.4 \%$, and rheumatoid arthritis $0.9 \%[1,7]$. A study done in Turkey did not find a significant difference between disease severity and personal or family history of autoimmune disease in adult and paediatric patients [8]. However a study from Taiwan found an association between comorbid conditions and age of onset of AA [9]. In 10-15\% patients, the nails are involved.

Poor prognostic factors are, childhood onset atopy (personal or first-degree relatives), ophiasis pattern, extensive involvement, onychodystrophy and Down's syndrome $[1,3,4,9]$. Local steroids (topical or intralesional) are effective in treatment of localised AA [10]. Systemic steroids and topical minoxidil lotionare effective in severe disease [11, 12].

This study was conducted to assess the demographic pattern, clinical presentation, and therapeutic response of alopecia areata in a Sri Lankan population.

\section{Methods}

An observational study was conducted at dermatology units at Teaching Hospital, Anuradhapura ( $\mathrm{n}=154$, duration 18 months), General Hospital Chilaw ( $\mathrm{n}=107$, duration 18 months) and Base Hospital, Homagama $(\mathrm{n}=29$, duration 6 months). The total study period was from July 2008 to July 2013.

Demographic data, ethnicity, family history, associated diseases such as other autoimmune diseases, atopy, and eczema were recorded. Clinical type of lesion was categorised as alopecia areata (AA), alopecia totalis (AT), alopecia universalis (AU), ophiasis pattern or reverse ophiasis pattern. Patients were examined for the site, size and number of lesions and for associated nail changes such as pitting, trachyonychia, and longitudinal ridging.

Department of Dermatology, Base Hospital, Homagama, Sri Lanka.

Correspondence: RRR, e-mail: <ranthilaka37@yahoo.com>. Received 7 October 2013 and revised version accepted 4 June 2014. Competing interests: none declared. 
Treatment was decided according to the age of the patient, severity and the site of the lesion. Hair loss involving $10 \%$ or less of scalp area was treated with intralesional triamcinolone acetonidewith or without topical steroids. Those who could not tolerate pain due to intralesional injections were treated with topical steroids with or without systemic therapy. When there was hair loss involving more than $10 \%$ of scalp area, systemic therapy was added. Oral dexamethasone 0.5-3 mg twice weekly pulses and levimasole 40-120 mg twice weekly pulses were used depending on the severity of the disease and the age of the patient. When there was baldness involving more than $30 \%$ scalp area, alopecia totalisor alopecia universalis, $5 \%$ minoxidil lotion application was applied on the scalp 1-2 times a day. Pregnant or lactating women and the participants with a history of cardiac, renal or hepatic diseases were not treated with systemic therapy.

\section{Results}

\section{Description of the sample}

A total of 290 adult patients were included in the study (Table 1). Children were excluded from the study. Majority were Sinhalese. More males were affected than females, male: female ratio was 1.3: 1. Majority (61.3\%) were aged 20 to 35 years. The mean age at presentation was 31.8 years (median 31; range 18 to 65 years). Duration of disease at presentation varied from one week to seven years, (mean $=6$ months; median $=1.5$ months). Majority presented within one month of onset of disease.

\section{Clinical presentation}

Table 1. Socio demographic data of participants

\begin{tabular}{lc}
\hline & Number* $\%$ ) \\
\hline Age at presentation (years) & \\
$18-19$ & $17(6.7)$ \\
$20-35$ & $154(61.3)$ \\
$36-49$ & $65(25.8)$ \\
$50-65$ & $15(6.0)$ \\
& \\
Age at onset (years) & \\
$11-17$ (juvenile onset) & $5(2.3)$ \\
$18-35$ & $133(63.3)$ \\
$36-49$ & $59(28.0)$ \\
50 and above (late onset) & $12(6.0)$ \\
& \\
Disease duration at presentation & $124(46.8)$ \\
Within one month & $94(35.4)$ \\
$2-6$ months & $15(5.7)$ \\
$7-11$ months & $17(6.4)$ \\
$12-24$ months & $14(5.2)$ \\
3 years and more & \\
\hline
\end{tabular}

*Totals may vary due to missing data
AA was the commonest clinical presentation, while alopecia universalis, alopecia totalis, ophiais pattern and reverse ophiasis pattern were seen in the descending order. (Table 2). Scalp was the commonest single site involved in adults with AA. Nearly one third $(29.6 \%)$ of men had lesions in the beard area only and $8.7 \%$ had lesions on multiple sites (scalp, beard, eye brows and mustache). Number of lesions in individual patients varied from one to eleven; $41 \%$ had a single lesion. More than $80 \%$ had mild AA involving $10 \%$ or less of scalp area. In $52(18 \%)$ patients alopecia was total, universal or extensive involving more than $10 \%$ scalp area.

Table 2. Clinical manifestations

\begin{tabular}{|c|c|}
\hline & Number* $(\%)$ \\
\hline \multicolumn{2}{|l|}{ Clinical presentations and severity } \\
\hline \multicolumn{2}{|l|}{ Mild alopecia areata } \\
\hline ( $\leq 10 \%$ scalp area involved) & $238(82.0)$ \\
\hline \multicolumn{2}{|l|}{$\begin{array}{l}\text { Extensive alopecia areata } \\
(\geq 10 \% \text { scalp area involved })\end{array}$} \\
\hline Alopecia totalis & $3(1.0)$ \\
\hline Alopecia universalis & $10(3.4)$ \\
\hline Ophiasis pattern & $3(1.0)$ \\
\hline Reverse ophiasis pattern & $1(0.4)$ \\
\hline \multicolumn{2}{|l|}{ Site of involvement } \\
\hline Only scalp & $169(70.7)$ \\
\hline Only beard area & $49(20.5)$ \\
\hline Multiple sites & $21(8.7)$ \\
\hline \multicolumn{2}{|l|}{$\begin{array}{l}\text { Number of lesions at presentation in } \\
\text { alopecia areata }\end{array}$} \\
\hline One lesion & $107(41.0)$ \\
\hline 2 or 3 lesions & $104(40.0)$ \\
\hline 4 to 11 lesions & $41(15.8)$ \\
\hline More than 11 lesions & $8(3.0)$ \\
\hline \multicolumn{2}{|l|}{ Associated diseases } \\
\hline Atopy, asthma or eczema & $60(21.0)$ \\
\hline Thyroid disease & $5(1.7)$ \\
\hline Vitiligo & $6(2.0)$ \\
\hline Rheumatoid arthritis & $1(0.3)$ \\
\hline Associated nail changes & $27(9.0)$ \\
\hline \multicolumn{2}{|l|}{ Relapses in patients with alopecia areata } \\
\hline First episode (no relapse) & $244(84.0)$ \\
\hline One relapse & $20(6.8)$ \\
\hline Two or more relapses & $27(9.0)$ \\
\hline
\end{tabular}

*Totals may vary due to missing data

Alopecia universalis $(\mathrm{n}=10)$ was present equally in men and women. Age at onset ranged from 17 to 55 years, $($ mean $=28.5$ years $)$. Duration of the present episode ranged from three months to five years. Eight patients had several relapses. Other than one patient whose brother 
had AA, others did not have a family history. Nail pitting was seen in one and atopy in two; none had associated autoimmune diseases.

Alopecia total is $(n=3)$ was observed in two men and one woman aged 19, 25 and 36 years. Both men were army soldiers while the woman was a house wife. Age at onset of AT was earlier (mean $=26.6$ years). All were first episode, there was no familial occurrence, there was no associated atopy or other autoimmune diseases. One patient had nail pitting.

Ophiasis pattern $(n=3)$ was observed in two women and man. Age at onset was 38, 42 and 17 years respectively. One had atopy; none had other associated autoimmune diseases, there were no nail changes or family history. Reverse ophiasis pattern was observed in a 38 year old woman, whose age at onset was 27 years. She had eczema and nail pitting but no other autoimmune diseases or family history of AA.

\section{Age of onset}

Age of onset ranged from 11 to 65 years, (mean= 32). There were three men and two women aged 18-24 years, who had juvenile-onset AA. The age of onset was 11, 12 and 17 years. Extensive disease was seen in three $(60 \%)$. Four $(80 \%)$ had several relapses over one to seven years. None had other associated diseases, nail changes or a family history. Twelve patients (11 males and one female) had late onset AA, the age of onset was more than 50 years. Six $(50 \%)$ had a single patch and ten $(83.3 \%)$ had less than $10 \%$ of the scalp area involved. Eczema was seen in two, thyroid disease in one, none had immediate family members with AA.

\section{Associated factors}

Atopy, eczema or asthma was present in 60 (21\%). Mean age at onset in this group was 32 years (median= 33 years; range $19-57$ years) $78 \%$ had mild disease and 50 $(83 \%)$ were in the first episode. Associated autoimmune diseases were present in $12(4 \%)$; vitiligo in six $(2 \%)$, thyroid disease in five $(1.7 \%)$ and rheumatoid arthritis in one $(0.3 \%)$. Mean age at onset was 34.4 years (median= 33 years; range 23-54 years). All had mild disease.

Thirty two $(11 \%)$ had a family history in brother $(n=15)$, sister $(n=4)$, mother $(n=4)$, father $(n=3)$, cousin $(n=2)$, son $(n=1)$, maternal uncle $(n=2)$ or grandmother $(n=1)$. Although onset of disease in this group was earlier ( mean $=30.5$ years; median $=30$ years; range $19-43$ years) $94 \%$ had mild disease, and only one third (37.5\%) had experienced relapses. Nail changes consisting of pitting, trachyo-nychia, and longitudinal ridging, were seen in $27(9 \%)$ and were more frequent in those with extensive disease (52\%). Nail pitting was the commonest association observed. Mean age at onset in this group was 31.5 years; range 18-49 years.

\section{Therapeutic response}

Eight patients who refused intralesional steroids for single lesion smaller than $3 \mathrm{~cm}$ were treated with topical clobetasol ointment for four months, and one showed spontaneous re-growth. Most (70\%) were treated with monthly intralesional steroids. Nearly one third were cured after a single injection, and further $31.7 \%$ were cured after two injections (Table 3). Remaining 9.7\% needed 3-5 injections and in addition oral therapy. Atrophy $(n=9)$ and scarring $(n=6)$ were the side effects observed with intralesional steroids.

Table 3. Therapeutic response

\begin{tabular}{lc}
\hline & Number* $\%)$ \\
\hline Cured with topical steroids alone & $8(2.7)$ \\
$\begin{array}{l}\text { Number of patients treated with } \\
\text { intralesional steroids } \\
\quad \text { Cured after a single injection } \\
\quad \text { Cured after two injections }\end{array}$ & $203(70.0)$ \\
& $83(28.6)$ \\
$\begin{array}{l}\text { Treated with oral dexamethasone } \\
\text { mini pulse }\end{array}$ & $92(31.7)$ \\
Treated with minoxidil lotion & $82(28.3)$ \\
\hline
\end{tabular}

Eighty two (28\%) were given oral dexamethasone mini pulse with or without topical 5\% minoxidil lotion $(\mathrm{n}=42,14.5 \%)$ for 12 months or more depending on the severity of the disease. Treatment was tailed-off over another 6 months. With combination therapy patients had hair growth in more than $50 \%$ of the scalp area within 6-8 months. Topical minoxidil was withheld in one patient who developed irritation after application. With pulsed dexamethasone therapy systemic side effects such as weight gain, increased urination and dizziness were observed in one patient with alopecia universalis who was treated for sixteen months.

Default rate was low $(6.6 \%)$ in those with mild disease. Patients with severe and prolonged disease had higher default rates $(25 \%)$.

\section{Discussion}

Our data confirms that AA is predominantly a disease of the young. Majority had mild disease involving $10 \%$ or less of scalp area. Disease severity could be predicted by age at onset. Patients with juvenile onset had severe disease with many relapses and resistance to therapy, while late onset AA, was characterised by female preponderance and mild disease activity. Prevalence of AA among first degree relatives was $11 \%$ which was similar to previous findings of $10-20 \%$. Those with a 
family history had earlier onset and majority (93\%) had mild disease. Previous studies have reported that 20-30\% of patients with AA have atopy which was similar to our study [7]. Age at onset and severity of the disease in patients with atopy was similar to those without atopic disease. Association between severe AA and familial auto-immunity has been suggested [7]. In our study 12 (4\%) had associated autoimmune diseases. In this group, age of onset was similar to the non-autoimmune group, and eleven out of twelve had mild disease.

Nail changes in our patients $(9 \%)$ were less than that reported previously (10-15\%). Fifty two percent of our patients with nail changes had extensive disease similar to previous reports $[1,2]$.

Intralesional triamcinolone acetonide was the firstline treatment for limited alopecia areata and was well tolerated with minimal side effects. Of the 203 patients treated with intralesional steroids, only $6 \%$ had significant local side effects. Studies report that combination therapy of oral dexamethasone mini pulse and topical minoxidil lotion is effective on severe recalcitrant AA [11]. These were well tolerated in our patients, only one patient developed side effects with systemic steroids, and one patient developed irritation with topical minoxidil. We did not use oral minoxidil. Although patients responded to combination therapy, in those with severe disease, discontinuation of treatment was associated with relapses. Patients with mild AA had good response to therapy, and good compliance. Irregular clinic visits and high default rates were evident in patients with extensive disease.

In conclusion, extensive involvement, juvenile onset, and associated nail changes were identified as poor prognostic factors of AA. Atopy was the most frequent association but we did not find an association between atopy and severity of disease. Combinations of oral dexamethasone mini pulse with topical minoxidil is effective and safe in extensive disease.

\section{Acknowledgements}

I thank Dr. Indu Seneviratne, medical officer at Teaching Hospital Anuradhapura for assisting in data collection.

\section{References}

1. Tan E, Tay YK, Goh CL, Chin GY. The pattern and profile of alopecia areata in Singapore - a study of 219 Asians. International Journal of Dermatology 2002; 41: 748-53.

2. Sharma VK, Dawn G, Kumar B. Profile of alopecia areata in Northern India. International Journal of Dermatolology 1996; 35: 22-7.

3. Kavak A, Yeşildal N, Parlak AH, Gökdemir G, Aydođan I, Anul H, Baykal C. Alopecia areata in Turkey: demographic and clinical features. Journal of the European Academy of Dermatology and Venereology 2008; 22: 977-81.

4. De Waard-van der Spek FB, Oranje AP, De Raeymaecker DM, Peereboom-Wynia JD. Juvenile versus maturity-onset alopecia areata - a comparative retrospective clinical study. Clinical and Experimental Dermatology 1989; 14: 429-33.

5. Alexis AF, Dudda-Subramanya R, Sinha AA. Alopecia areata: autoimmune basis of hair loss. European Journal of Dermatology 2004; 14: 364-70.

6. Thomas EA, Kadyan RS. Alopecia areata and autoimmunity: a clinical study. Indian Journal of Dermatology 2008; 53: 70-4.

7. Goh C, Finkel M, Christos PJ, Sinha AA. Profile of 513 patients with alopecia areata: associations of disease subtypes with atopy, autoimmune disease and positive family history. Journal of the European Academy of Dermatology and Venereology 2006; 20: 1055-60.

8. Chu SY, Chen YJ, Tseng WC, et al. Co-morbidity profiles among patients with alopecia areata: the importance of onset age, a nationwide population-based study. Journal of the American Academy of Dermatology 2011; 65: 949-56.

9. Kuldeep C, Singhal H, Khare AK, Mittal A, Gupta LK, Garg A. Randomized comparison of topical betamethasone valerate foam, intralesional triamcinolone acetonide and tacrolimus ointment in management of localized alopecia areata. International Journal of Trichology 2011; 3: 20-4.

10. Deshpande D, Dhurat R, Saraogi P, Mishra S, Nayak C. Extensive alopecia areata: not necessarily recalcitrant to therapy! International Journal of Trichology 2011; 3: 80-3.

11. Nakajima T, Inui S, Itami S. Pulse corticosteroid therapy for alopecia areata: study of 139 patients. Dermatology 2007; 215: $320-4$. 